\title{
Antiretroviral therapy programmes in resource limited settings
}

In the first paragraph of the online version of this editorial by Carlos del Rio and Wendy Armstrong (BMJ 2011;343:d6853, doi:10.1136/bmj.d6853), we refer to two linked articles. The first author of the second linked article (cited as reference 2) is spelt wrongly in reference 2 and in the sixth paragraph of the article. The correct spelling is Kahn (not Khan).

Cite this as: BMJ 2011;343:d7467

๑ BMJ Publishing Group Ltd 2011 